\title{
Molecular Epidemiology of Mycobacterium tuberculosis Complex Strains in Urban and Slum Settings of Nairobi, Kenya
}

\author{
Glennah Kerubo ${ }^{1,2, *}$, Perpetua Ndungu ${ }^{3}$, Yassir Adam Shuaib ${ }^{1,4} \oplus^{(}$, Evans Amukoye $^{5}{ }^{(}$, Gunturu Revathi $^{6}$, \\ Susanne Homolka ${ }^{1}$, Samuel Kariuki ${ }^{7,+}+$, Matthias Merker ${ }^{1,8,9,+}$ and Stefan Niemann ${ }^{1,8, *,+}$
}

1 Molecular and Experimental Mycobacteriology, Research Center Borstel, 23845 Borstel, Germany; vet.aboamar@gmail.com (Y.A.S.); shomolka@fz-borstel.de (S.H.); mmerker@fz-borstel.de (M.M.)

2 School of Medicine, Kenyatta University, Nairobi 43844-00100, Kenya

3 Jomo Kenyatta University of Agriculture and Technology, Nairobi 62000-00200, Kenya; ndunguperpetual@gmail.com

4 College of Veterinary Medicine, Sudan University of Science and Technology, Khartoum North 13321, Sudan

5 Centre for Respiratory Disease Research, Kenya Medical Research Institute, Nairobi 54840-00200, Kenya; amukoye@gmail.com

6 Aga Khan University Hospital, Nairobi 30270-00100, Kenya; gunturu.revathi@aku.edu

7 Centre for Microbiology Research, Kenya Medical Research Institute, Nairobi 54840-00200, Kenya; samkariuki2@gmail.com

8 German Center for Infection Research (DZIF), Partner Site Hamburg-Lübeck-Borstel-Riems, 23845 Borstel, Germany

9 Evolution of the Resistome, Research Center Borstel, 23845 Borstel, Germany

* Correspondence: glenakerubo@gmail.com (G.K.); sniemann@fz-borstel.de (S.N.)

+ These authors contributed equally to this work.

Citation: Kerubo, G.; Ndungu, P.;

Shuaib, Y.A.; Amukoye, E.; Revathi,

G.; Homolka, S.; Kariuki, S.; Merker,

M.; Niemann, S. Molecular

Epidemiology of Mycobacterium

tuberculosis Complex Strains in Urban and Slum Settings of Nairobi, Kenya. Genes 2022, 13, 475. https://doi.org/ 10.3390/genes13030475

Academic Editor: Silvia Turroni

Received: 7 February 2022

Accepted: 2 March 2022

Published: 8 March 2022

Publisher's Note: MDPI stays neutral with regard to jurisdictional claims in published maps and institutional affiliations.

Copyright: (c) 2022 by the authors. Licensee MDPI, Basel, Switzerland. This article is an open access article distributed under the terms and conditions of the Creative Commons Attribution (CC BY) license (https:// creativecommons.org/licenses/by/ $4.0 /)$.

\begin{abstract}
Kenya is a country with a high tuberculosis (TB) burden. However, knowledge on the genetic diversity of Mycobacterium tuberculosis complex (MTBC) strains and their transmission dynamics is sparsely available. Hence, we used whole-genome sequencing (WGS) to depict the genetic diversity, molecular markers of drug resistance, and possible transmission clusters among MTBC strains in urban and slum settings of Nairobi. We analyzed 385 clinical MTBC isolates collected between 2010 and 2015 in combination with patients' demographics. We showed that the MTBC population mainly comprises strains of four lineages (L1-L4). The two dominating lineages were L4 with $55.8 \%(n=215)$ and L3 with $25.7 \%(n=99)$ of all strains, respectively. Genome-based cluster analysis showed that $30.4 \%(117 / 385)$ of the strains were clustered using a $\leq 5$ single-nucleotide polymorphism (SNP) threshold as a surrogate marker for direct patient-to-patient MTBC transmission. Moreover, $5.2 \%(20 / 385)$ of the strains were multidrug-resistant (MDR), and $50.0 \%(n=10)$ were part of a genome-based cluster (i.e., direct MDR MTBC transmission). Notably, $30.0 \%(6 / 20)$ of the MDR strains were resistant to all first-line drugs and are part of one molecular cluster. Moreover, TB patients in urban living setting had 3.8 times the odds of being infected with a drug-resistant strain as compared to patients from slums $(p$-value $=0.002)$. Our results show that L4 strains are the main causative agent of TB in Nairobi and MDR strain transmission is an emerging concern in urban settings. This emphasizes the need for more focused infection control measures and contact tracing of patients with MDR TB to break the transmission chains.
\end{abstract}

Keywords: tuberculosis; Mycobacterium tuberculosis; whole-genome sequencing; molecular epidemiology; Nairobi

\section{Introduction}

Tuberculosis (TB) is still a major cause of morbidity and mortality worldwide, especially in Sub-Saharan Africa [1-3]. In 2020, TB was responsible for 9.9 million new cases, of which $25 \%$ were in the World Health organization (WHO) African region [1]. Kenya is among the highest TB burden countries with an incidence of 259/100,000 population, which 
is twice as much as the global average [1-3]. The total number of new and relapse TB case notifications was 71,646 [1]. The HIV/AIDS epidemic and the emergence of drug-resistant Mycobacterium tuberculosis complex (MTBC) strains complicated the effective control of TB in the country $[4,5]$. Additionally, the emergence of SARS-CoV-2 is expected to have a longlasting impact leading to an increase in the burden of TB in Kenya due to the disruption of TB health care services in 2020 [6,7]. Moreover, the lack of resources to carry out proper and definitive diagnosis of TB fuels the country's TB epidemic as TB diagnosis is solely performed using smear microscopy in Kenya. This technique has a variable sensitivity and does not allow for resistance prediction or differentiation between infections due to MTBC and non-tuberculous mycobacteria (NTM) $[8,9]$. TB cases are therefore treated using empirical treatment which may foster the development and transmission of resistant MTBC strains by long periods of infectivity [10].

In Kenya, knowledge on genetic drug resistance markers of MTBC strains is inadequate, especially in potential transmission hotspots, such as the capital Nairobi. Molecular epidemiological studies have suggested that transmission is a major driver of drug susceptible and drug resistant TB epidemics, not only in Eastern Europe but also in Africa [10-13]. Indeed, MTBC transmission is one of the major constraints that hinder TB control, particularly in poor settings (e.g., slums) where the risk of transmission is high due to overcrowding $[1,13]$. Evidence has disclosed high TB prevalence in slums in Kenya [14] and other African countries, such as South Africa $[15,16]$. Furthermore, patients in slums may have limited access to TB treatment centers.

Here, we collated MTBC strains from two patient cohorts in Nairobi to analyze differences in the genetic diversity, transmission dynamics, and molecular drug resistance profiles of MTBC strains circulating in slum and urban settings.

\section{Materials and Methods}

\subsection{Study Design and Setting}

We enrolled self-presented presumptive TB patients in this cross-sectional study who had respiratory symptoms and were smear-positive from 13 randomly selected health centers located in Nairobi and its surroundings (Figure S1, Table S1). The first cohort $(n=204)$ was enrolled between May 2010 and May 2013, and the second cohort $(n=275)$ was enrolled from September 2014 to May 2015. Three of the health care centers selected are TB referral centers (Table S1), of which one center is located in slums (i.e., highly populated or informal settlements with weak building quality, inadequate housing or very small living spaces, and squalid conditions which are often associated with poverty) and serves up to 200 patients per week. Each patient was required to give a spot (i.e., collected at the time of first visit of the patient to clinic/laboratory) and an early morning sputum sample for analysis. Both spot and early morning samples were pooled and used for microbiological analysis. Demographic data were gathered using a structured questionnaire, and each recruited patient was assigned a unique personal identifier that was used throughout the study period.

\subsection{Laboratory Procedures}

\subsubsection{Sputum Decontamination, Mycobacterial Culture, and DNA Extraction}

All specimens were processed and decontaminated following the standard $\mathrm{N}$-acetyl-Lcysteine-sodium hydroxide (NALC-NaOH) method for digestion, decontamination, and concentration [17]. The sediment was resuspended in about 1 to $2 \mathrm{~mL}$ phosphate buffer (pH 6.8) and mixed thoroughly. A smear was prepared for acid-fast staining $[9,18]$.

Mycobacterial cultures were performed by adding $0.25 \mathrm{~mL}$ of the suspended decontaminated samples into the BACTEC MGIT 960 (Becton Dickinson, Franklin Lakes, NJ, USA) and onto solid Löwenstein-Jensen (LJ) culture media [19]. 
DNA extraction from mycobacterial colonies was carried out using the cetyl trimethylammonium bromide (CTAB) method as described by Somerville et al. [20]. The DNA was reconstituted with molecular grade water and stored at $-20^{\circ} \mathrm{C}$ until it was sent to the Molecular and Experimental Mycobacteriology Laboratory, Research Centre Borstel in Germany, where molecular characterization of the MTBC strains was conducted.

\subsubsection{Whole-Genome Sequencing}

DNA libraries for whole-genome sequencing (WGS) were prepared with the Nextera (XT) kit from Illumina (San Diego, USA) according to the manufacturer's instructions [21]. Pooled DNA libraries were then loaded into NextSeq Reagent cartridges for sequencing on a NextSeq system (Illumina, San Diego, CA, USA). Resulting sequencing reads were submitted to the European Nucleotide Archive under the project accession number PRJEB50767 and subsequently mapped to the H37Rv reference genome (GenBank ID: NC_000962.3) by Burrows-Wheeler alignment (BWA) tool aiming for a minimum of 50-fold average genome-wide coverage [22,23]. We considered single nucleotide polymorphisms (SNPs) with at least 4 reads in both forward and reverse orientation, 4 reads calling the allele with at least a Phred score of 30, and 75\% allele frequency for a concatenated sequence alignment. SNP positions that had reliable base call (as described above) in at least $95 \%$ of the strains were concatenated to a sequence alignment. SNPs from repetitive regions were excluded, including those which occurred within a window of 12 base pairs in neighboring strains [23].

A web tool was used for detecting isolates that harbored more than one phylogenetic lineage (i.e., mixed infections or laboratory contaminations) [24]. These samples/isolates were not considered in subsequent analysis.

\subsection{Data Analysis}

\subsubsection{Phylogenetic Reconstruction and WGS-Based Cluster Inference}

On the basis of concatenated sequence data, the most suitable substitution model for the dataset was implemented using the maximum likelihood ratio test (Jmodel test) [25]. A maximum likelihood tree was calculated based on the concatenated sequence alignment using FastTree [26] with a general time-reversible (GTR) substitution model (best according to Jmodel test), 1000 resamples, and $\gamma 20$ likelihood optimization to account for the rate of heterogeneity among sites. The phylogenetic tree was inspected and rooted with FigTree software (Institute of Evolutionary Biology, University of Edinburgh, Edinburgh, Scotland). Graphical presentation was performed with Evolview [27]. The concatenated sequence data were also used to calculate maximum parsimony trees using BioNumerics software version 7.6 (Applied Maths, BioMérieux, Sint-Martens-Latem, Belgium). Genome clusters were defined by grouping all sequenced isolates within a maximum distance of $\leq 12$ and $\leq 5$ SNPs between one strain and the neighboring strain [28,29].

\subsubsection{Molecular Drug Resistance Prediction}

Genes associated with drug resistance mechanisms were individually investigated and not considered for phylogenetic approaches [24].

The SNP dataset was examined for known resistance-conferring mutations against first-line and second-line antibiotics [24]. In brief, for rifampicin (RIF) resistance, mutations that confer resistance on the $r p o B$ gene were analyzed. Other RNA-polymerase genes, i.e., $r p o A$ and $r p o C$, were analyzed with regard to putative compensatory mutations. We also investigated $k a t G$, inh $A, n d h$, and $f a b G 1$ genes for mutations that confer resistance to isoniazid (INH). We screened $p n c A$ and $r p s A$ genes for mutations conferring resistance to pyrazinamide (PZA), and $e m b B, e m b C$, and $e m b A$ genes for mutations associated with ethambutol (EMB) resistance. In the case of streptomycin (STR) resistance, the $r p s L$, rrs, and gidB genes were investigated for mutations conferring resistance. For isolates that were MDR (i.e., resistance towards INH and RIF), genes conferring resistance against secondline drugs were examined. The gyrA and gyrB genes were investigated for resistance 
against fluoroquinolones (FQs), while the rrs gene was studied for resistance against aminoglycosides. In addition, $t l y A$ and $t h y A$ genes were analyzed for capreomycin (CAP) and para-aminosalicylic acid (PAS) resistance, respectively. For ethionamide (Eto) resistance, ethA and fabG1 genes were studied [24].

\subsubsection{Statistics}

Demographic data were entered, cleaned, and analyzed using the SPSS statistical software package, version 20 (SPSS Inc., Chicago, IL, USA). Chi-square test and Fisher exact test were used to compare categorical variables. All variables were tested using logistic regression analysis. A $p$-value $\leq 0.05$ was considered significant.

\subsection{Ethical Considerations}

This study was approved by the Scientific and Ethical Review Unit of Kenya Medical Research Institute (SSC Protocol No. 2830). All patients recruited into the study were required to sign a written informed consent for collection and analysis of samples and demographic and clinical data. If the patient was illiterate or less than 18 years old, a caretaker was requested for consent. All the information collected from cases was kept confidential. All patient identifiers were removed prior to data analysis. During the study, any diagnosed TB cases (i.e., smear-positive) were registered at the health centers and treated according to the National Tuberculosis and Leprosy Programme (NTLP) guidelines [30].

The NTLP guidelines state that all patients who have not been on TB therapy previously should have a two-month initial phase of treatment consisting of INH, RIF, PZA, and EMB followed by a continuation phase of EMB and INH for six months or INH and RIF for four months [30]. For retreatment TB patients, the intensive phase is three months of daily injections of STR and swallowing of INH, RIF, PZA, and EMB, followed by five months of continuation phase with INH, RIF, and EMB [30].

\section{Results}

\subsection{Study Population}

A total of 479 sputum samples were collected from smear-positive patients in health centers in Nairobi and its surroundings (Figure S1). Based on hospital records, the recruited patients comprised 10-20\% of all patients who received a diagnosis of TB during the study period.

After excluding individuals with missing data, we found that the overall mean age of the investigated patients was $31.7 \pm 8.3$ years. Moreover, we observed that most of the enrolled individuals $(64.4 \%, 246 / 382)$ were males and were newly diagnosed TB cases $(85.3 \%, 326 / 382)$. Most of the study cases were from health centers located in non-slum areas (i.e., urban or developed areas with well-constructed houses, commercial buildings, roads, and bridges) $(55.8 \%, 215 / 385)$, while $44.2 \%(170 / 385)$ were from health centers located in slums (informal settlements) (Table S1).

Out of all collected sputum specimens, $48 / 479$ (10.0\%) were either culture-negative or contaminated (Figure 1). Furthermore, 31 isolates were subsequently excluded from the analysis due to quality issues or the identification of more than one strain $(n=15)$ in the DNA isolate. The final analysis comprised 385/479 (80.4\%) WGS datasets, each representing one isolate per patient (Figure 1). 


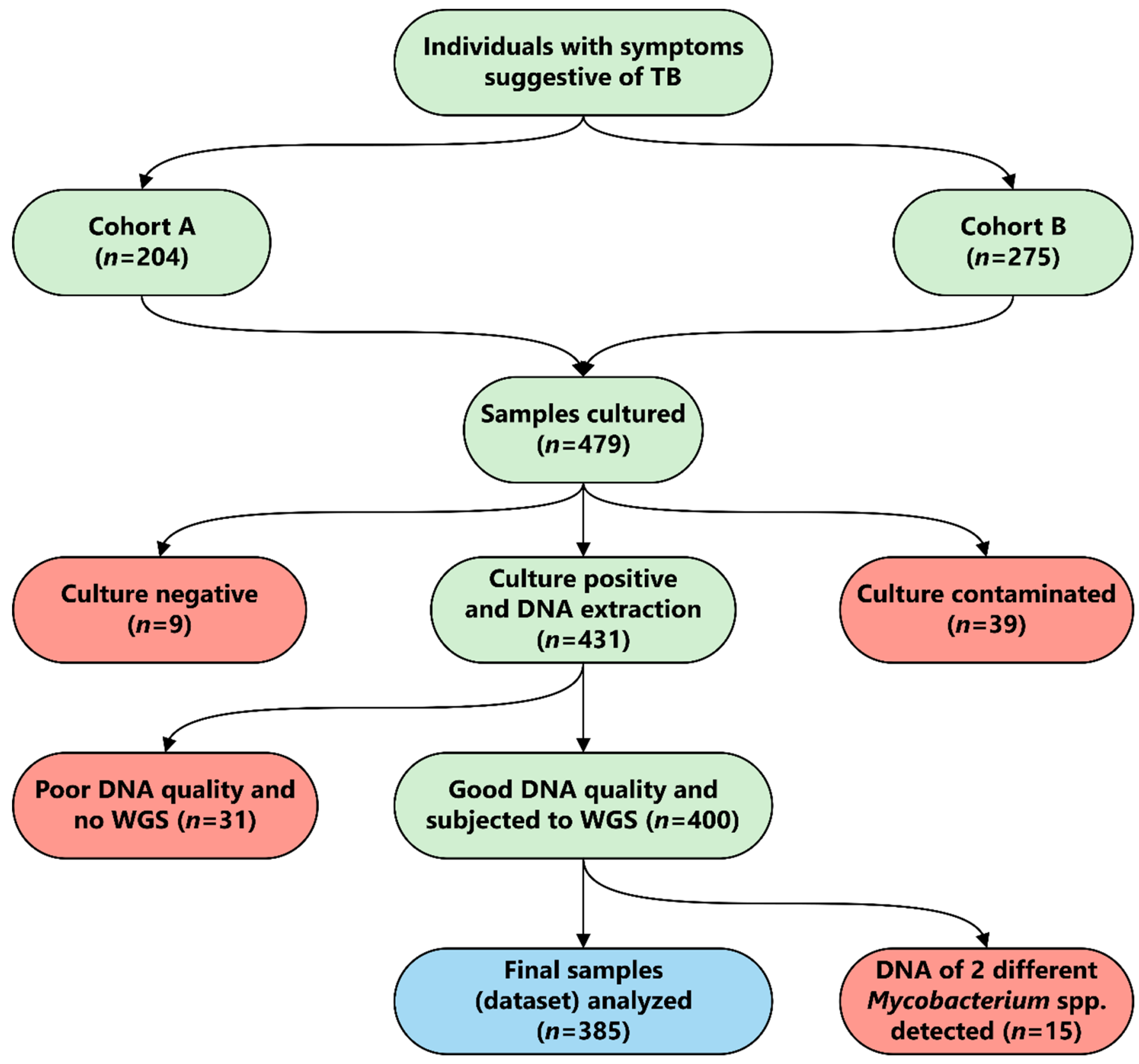

Figure 1. Workflow for the study "Molecular epidemiology of Mycobacterium tuberculosis complex strains in urban and slum settings of Nairobi, Kenya". TB = tuberculosis, Cohort A = patients enrolled in 2010, Cohort B = patients enrolled in 2014-2015, WGS = whole-genome sequencing, spp. = species.

Comparison of the two patient cohorts (i.e., 2010 cohort vs. 2014-2015 cohort) revealed significant differences between the proportions of drug-susceptible and drug-resistant strains $\left(X^{2}=25.3, p\right.$-value $\left.\leq 0.001\right)$, the proportions of clustered and not clustered strains based on $\leq 5$ SNP threshold $\left(X^{2}=14.2, p\right.$-value $\left.\leq 0.001\right)$, and the proportions of male and female sex $\left(X^{2}=4.97, p\right.$-value $\left.=0.026\right)$ after excluding individuals with missing information from the analysis (Table 1). Furthermore, the proportions of patients residing in urban and slum living conditions differed significantly between the two cohorts $\left(\mathrm{X}^{2}=178.2\right.$, $p$-value $\leq 0.001)$. While in the 2010 cohort $91.7 \%(166 / 181)$ of the patients came from urban areas, only $24.0 \%(49 / 204)$ of the patients from the 2014-2015 cohort were living in an urban setting (Table 1). 
Table 1. Comparison of the two patient cohorts investigated by using chi-square test in Nairobi, Kenya (2010-2015).

\begin{tabular}{|c|c|c|c|c|c|}
\hline Variable & No. & Cohort A & Cohort B & $x^{2}$ & $p$-Value \\
\hline Lineage & & & & 7.70 & 0.103 \\
\hline L1 (EAI) & 14 & $9(5.0 \%)$ & $5(2.5 \%)$ & & \\
\hline L2 (Beijing) & 56 & $30(16.6 \%)$ & $26(12.7 \%)$ & & \\
\hline L3 (Delhi/CAS) & 99 & $52(28.7 \%)$ & $47(23.0 \%)$ & & \\
\hline L4 (Euro-American) & 215 & $89(49.2 \%)$ & $126(61.8 \%)$ & & \\
\hline M. bovis & 1 & $1(0.5 \%)$ & $0(0.00 \%)$ & & \\
\hline DST & & & & 25.3 & $<0.001^{\mathrm{a}}$ \\
\hline Susceptible & 348 & $151(83.4 \%)$ & 197 (96.6\%) & & \\
\hline MDR & 20 & $20(11.1 \%)$ & $0(0.00 \%)$ & & \\
\hline Non-MDR & 17 & $10(5.5 \%)$ & $7(3.4 \%)$ & & \\
\hline d12 & & & & 3.08 & 0.079 \\
\hline Clustered & 218 & $111(61.3 \%)$ & 107 (52.5\%) & & \\
\hline Not clustered & 167 & $70(38.7 \%)$ & $97(47.5 \%)$ & & \\
\hline d5 & & & & 14.2 & $<0.001^{a}$ \\
\hline Clustered & 117 & $72(39.8 \%)$ & $45(22.1 \%)$ & & \\
\hline Not clustered & 268 & $109(60.2 \%)$ & $159(77.9 \%)$ & & \\
\hline TB history & & & & 0.11 & 0.747 \\
\hline New & 326 & $152(84.0 \%)$ & $174(85.3 \%)$ & & \\
\hline Retreatment & 56 & $28(15.5 \%)$ & $28(15.5 \%)$ & & \\
\hline $\mathrm{NA}^{*}$ & 3 & $1(0.5 \%)$ & $2(1.0 \%)$ & & \\
\hline Age group & & & & 0.11 & 0.917 \\
\hline$\leq 30$ & 166 & $63(34.8 \%)$ & 103 (50.5\%) & & \\
\hline$>30$ & 161 & $62(34.3 \%)$ & $99(48.5 \%)$ & & \\
\hline NA * & 58 & $56(30.9 \%)$ & $2(1.0 \%)$ & & \\
\hline Sex & & & & 4.97 & $0.026^{a}$ \\
\hline Male & 246 & $105(58.0 \%)$ & 141 (69.1\%) & & \\
\hline Female & 136 & $75(41.4 \%)$ & $61(29.9 \%)$ & & \\
\hline NA * & 3 & $1(0.6 \%)$ & $2(1.0 \%)$ & & \\
\hline Sampling area & & & & 178.2 & $<0.001^{a}$ \\
\hline Urban & 215 & $166(91.7 \%)$ & $49(24.0 \%)$ & & \\
\hline Slum & 170 & $15(8.3 \%)$ & $155(76.0 \%)$ & & \\
\hline Total & 385 & $181(47.0 \%)$ & $204(53.0 \%)$ & - & - \\
\hline
\end{tabular}

No. = number, $\mathrm{A}$ = cohort 2010, B = cohort 2014-2015, DST = drug susceptibility testing, MDR = simultaneous resistance towards isoniazid and rifampicin, non-MDR = resistance towards drugs other than isoniazid and rifampicin, d12 = genetic distance of $\leq 12$ single-nucleotide polymorphisms (SNPs), d5 = genetic distance of $\leq 5$ SNPs, NA $=$ not available, ${ }^{*}=$ variable (individuals with missing data) excluded from analysis, ${ }^{a}=$ variable with a chi-square $p$-value of $\leq 0.05$.

\subsection{MTBC Population Structure}

We performed WGS successfully on 400 MTBC strains. We excluded 15 samples/datasets from the final analysis because of the identification of more than one strain and built a maximum likelihood phylogeny upon a concatenated sequence alignment comprising 18,167 SNPs to investigate the MTBC population structure using 385 strains (Figure 2). Phylogenetic lineages (Ls) were inferred from canonical SNPs specific for certain MTBC sublineages based on a recently introduced SNP barcode classification [31,32]. Strains of L4 (Euro-American super-lineage, $n=215 / 385,55.8 \%$ ) were predominant, followed by strains of L3 (Delhi/CAS, $n=99 / 385,25.7 \%$ ), strains of L2 (Beijing, $n=56 / 385,14.5 \%$ ), and strains of L1 (East African Indian, $n=14 / 385,3.6 \%$ ). There was one $M$. bovis strain identified (Figure 2). 


\subsection{1}

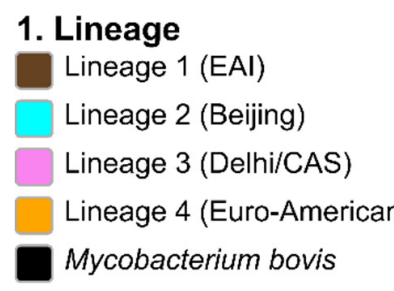

\section{Drug susceptibility}

Susceptible

Non-MDR

MDR

Pre-XDR

3. Sampling site Slum

Non-slum

\section{4. d12 SNP clusters \\ Clustered \\ Not clustered}

\section{5. d5 SNP clusters \\ Clustered}

Not clustered

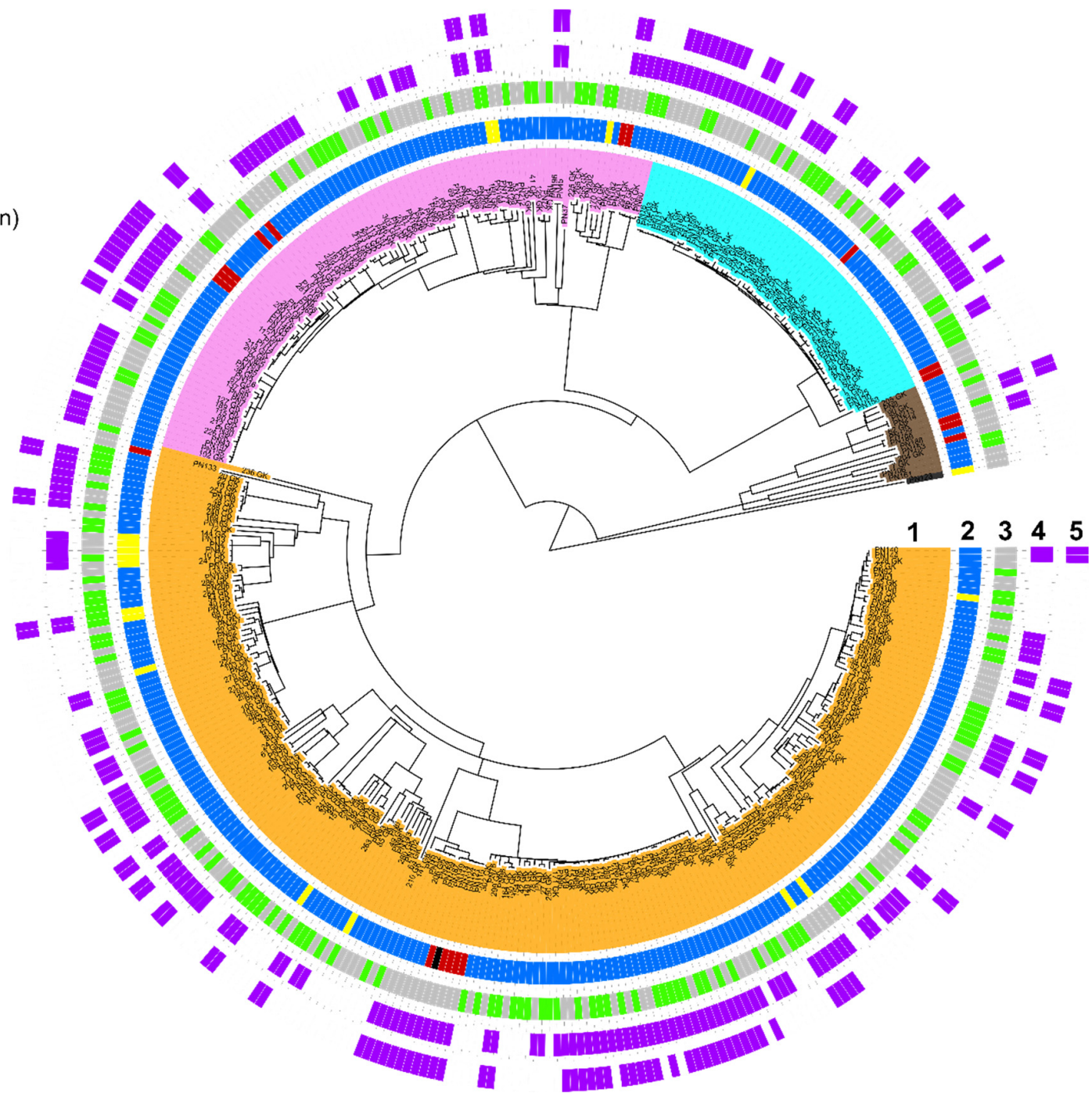

Figure 2. MTBC population structure in urban and slum areas of Nairobi, Kenya. Maximum likelihood tree based on 18,167 concatenated single-nucleotide polymorphisms (SNPs) using a general time-reversible substitution model. Colored bars code for (inner to outer ring) M. bovis and MTBC lineages (L1-4); genotypic DST results stratified to MDR, non-MDR, and fully susceptible; sampling location; and clustered and non-clustered strains (SNP distance $\leq 12$ and $\leq 5$ ). DST $=$ drug susceptibility testing, MDR = multidrug-resistant (simultaneous resistance towards isoniazid and rifampicin); Pre-XDR= pre-extensively drug-resistant (an MDR which is also resistant to any fluoroquinolone), MTBC $=$ Mycobacterium tuberculosis complex.

Strains of L4 (the Euro-American super-lineage) were further classified into several sublineages as described previously [31,32]. Strains of the Latin American Mediterranean (LAM) genotype $(n=70 / 215,32.5 \%)$ comprised the MTBC sublineages 4.3.2, 4.3.4.1, 4.3.4.2, and 4.3.4.2.1 and were most frequent, followed by S-type (4.4.1.1, $n=55 / 215,25.5 \%)$, Turkish (TUR) (4.2.2, $n=27 / 215,12.6 \%$ ), and Haarlem (4.1.2 and 4.1.2.1, $n=18 / 215,8.4 \%$ ) (Figure S2). Other sublineages identified include Kenya H37Rv-like $(4.8, n=13 / 215,6 \%)$, Uganda I (4.6.1.1, $n=7 / 215,3.2 \%)$, Uganda II (4.6.1.2 $n=10 / 215,4.6 \%)$, X-type (4.1.1.1, $n=7 / 215,3.2 \%)$, Cameroon $(4.6 .2 n=6 / 215,2.8 \%)$, Ghana $(4.1 n=1 / 215,0.5 \%)$, and undefined Euro-American (4.0, $n=1 / 215,0.5 \%$ ) (Figure S2).

\subsection{Genome-Based Drug Resistance Prediction}

Overall, 37 out of the 385 (9.6\%) MTBC isolates investigated were found to be resistant to at least one of the first-line drugs, of which $20(5.2 \%)$ were MDR. Monodrug resistance to 
INH, RIF, or PZA was observed in 15 (3.9\%), $1(0.3 \%)$, and $1(0.3 \%)$ of the MTBC strains, respectively (Table S1).

A detailed analysis of variants in resistance-associated genes revealed that $9.1 \%$ $(35 / 385)$ of the strains had mutations conferring resistance to INH, with the Ser315Thr in the $k a t G$ gene being the dominant observed mutation $(60.0 \%, 21 / 35)$ (Table 2). The $-15 \mathrm{c} / \mathrm{t}$ mutation in the fabG-inh $A$ promoter region was the second most dominant INH resistance determinant $(22.8 \%, 8 / 35)$. Variants in the rpoB gene that confer resistance to RIF were found in $21(5.5 \%)$ strains, with the Ser450Leu mutation being the most common $(52.3 \%$, 11/21) (Table 2). Moreover, mutations at codon 445 leading to His445Tyr, His445Arg, and His445Asp amino acid substitutions were observed in seven (33.3\%) strains.

Table 2. Detected mutations that mediate resistance to first-line anti-TB drugs in 37 resistant MTBC strains in urban and slum areas of Nairobi, Kenya (2010-2015).

\begin{tabular}{|c|c|c|c|c|}
\hline Drug & Gene & Mutation & Codon Change & Total \\
\hline \multirow{5}{*}{$\mathrm{INH}$} & \multirow{2}{*}{ katG } & Ser315Thr & $\operatorname{agc} / \mathrm{aCc}$ & 21 \\
\hline & & Ser315Arg & $\mathrm{agc} / \mathrm{aGa}$ & 2 \\
\hline & fabG1-inhA & $-15 \mathrm{c} / \mathrm{t}$ & $\mathrm{C} \rightarrow \mathrm{T}$ & 8 \\
\hline & \multirow{2}{*}{ inhA } & Leu203Leu & $\operatorname{ctg} / \operatorname{ct} A$ & 2 \\
\hline & & Ile194Thr & $\mathrm{atc} / \mathrm{aCc}$ & 2 \\
\hline \multirow{6}{*}{ RIF } & \multirow{6}{*}{$r p o B$} & Gln432Pro & $\mathrm{Caa} / \mathrm{cCa}$ & 2 \\
\hline & & His 445Tyr & $\mathrm{Cac} / \mathrm{Tac}$ & 5 \\
\hline & & His 445Arg & $\mathrm{Cac} / \mathrm{cGc}$ & 1 \\
\hline & & His445Asp & $\mathrm{Cac} / \mathrm{Gac}$ & 1 \\
\hline & & Ser450Leu & $\mathrm{Tcg} / \mathrm{tTg}$ & 11 \\
\hline & & Leu452Pro & $\mathrm{Ctg} / \mathrm{cCg}$ & 1 \\
\hline \multirow{3}{*}{ STR } & $r p s L$ & Lys43Arg & $\mathrm{Aag} / \mathrm{aGg}$ & 9 \\
\hline & $\operatorname{gidB}$ & Ala138Val & Gcg/gTg & 1 \\
\hline & $r r s$ & $513 \mathrm{a} / \mathrm{c}$ & $A \rightarrow C$ & 3 \\
\hline \multirow{4}{*}{ EMB } & \multirow{4}{*}{$e m b B$} & Met306Ile & Atg/atA & 7 \\
\hline & & Met306Val & Atg/Gtg & 2 \\
\hline & & Asp328Gly & Gat/gGt & 1 \\
\hline & & Asp354Ala & $\mathrm{Gac} / \mathrm{Aac}$ & 1 \\
\hline \multirow{6}{*}{ PZA } & \multirow{6}{*}{$p n c A$} & Gln10Pro & $\mathrm{cag} / \mathrm{cCg}$ & 1 \\
\hline & & His57Asp & $\mathrm{cac} / \mathrm{Gac}$ & 1 \\
\hline & & Asp63Gly & $\mathrm{gac} / \mathrm{gGc}$ & 2 \\
\hline & & Lsy96Thr & $\mathrm{aag} / \mathrm{aCg}$ & 6 \\
\hline & & Thr135Pro & $\mathrm{acc} / \mathrm{Ccc}$ & 1 \\
\hline & & Gln141_ & $\mathrm{cag} / \mathrm{tag}$ & 1 \\
\hline FQs & gyrA & Asp94Gly & $\mathrm{gac} / \mathrm{Cac}$ & 1 \\
\hline
\end{tabular}

$\overline{\mathrm{INH}}=$ isoniazid, $\mathrm{RIF}$ = rifampicin, STR, streptomycin, EMB = ethambutol, PZA = pyrazinamide, FQs = fluoroquinolones.

The most common mutation mediating STR resistances was the Lys43Arg $(69.2 \%$, $9 / 13)$ at the $r p s L$ gene, followed by the $513 \mathrm{a} / \mathrm{c}(23.0 \%, 3 / 13)$ mutation in the $r r s$ gene. Eleven strains were resistant to EMB, with Met306lle being the frequent mutation observed in the $e m b B$ gene $(63.6 \%, 7 / 11)$ (Table 2). The $p n c A$ gene was analyzed for mutations conferring resistance to PZA. The Lys96Thr mutation was found to be the most common $(50.0 \%, 6 / 12)$.

Only one strain was found with a fluoroquinolone resistance mediating mutation in the gyrA gene (Asp94Gly, 1/385, 0.3\%). This strain was hence defined as pre-extensively drug-resistant (XDR) which is an MDR and resistant to any fluoroquinolone. There were no resistance markers identified for other second-line anti-TB drugs.

When we compared the proportions of drug-resistant (i.e., any antibiotic resistance) and wild-type (fully susceptible) strains between sex groups, age groups, and TB history, we found no differences between male and female, $\leq 30$ and $>30$ age groups, and new 
and retreatment TB cases with a $p$-value of $\geq 0.247$ (Table 3). However, the proportion of infection with a drug-resistant strain was observed to be significantly associated with urban living conditions $(\mathrm{OR}=3.8,95 \%$ CI 1.62-8.83, $p$-value $=0.002)($ Table 3$)$. Notably, all MDR strains detected in this study were found in urban areas.

Table 3. Comparison of the proportions of drug-susceptible and drug-resistant strains across the sociodemographic variables and MTBC phylogenetic lineages by using logistic regression in Nairobi, Kenya (2010-2015).

\begin{tabular}{|c|c|c|c|c|c|}
\hline Variables & No. & $\begin{array}{c}\text { No. of Wild } \\
\text { Type }\end{array}$ & $\begin{array}{l}\text { No. of } \\
\text { Resistant } \\
(\%)\end{array}$ & $\begin{array}{c}\text { OR } \\
(95 \% \mathrm{CI})\end{array}$ & $p$-Value \\
\hline \multicolumn{6}{|l|}{ Gender } \\
\hline Male & 246 & 226 & $20(8.10)$ & Ref & \\
\hline Female & 136 & 120 & $16(11.8)$ & $\begin{array}{c}1.5 \\
(0.75-3.01)\end{array}$ & 0.247 \\
\hline $\mathrm{NA}^{*}$ & 3 & 2 & $1(33.3)$ & - & - \\
\hline \multicolumn{6}{|l|}{ Age } \\
\hline$\leq 30$ & 166 & 148 & $18(10.8)$ & Ref & \\
\hline$>30$ & 161 & 149 & $12(5.70)$ & $\begin{array}{c}1.5 \\
(0.70-3.24)\end{array}$ & 0.291 \\
\hline NA * & 58 & 51 & $7(12.1)$ & - & - \\
\hline \multicolumn{6}{|l|}{ Study area } \\
\hline Slum & 170 & 163 & $7(4.1)$ & Ref & \\
\hline Urban & 215 & 185 & $30(14.0)$ & $\begin{array}{c}3.8 \\
(1.62-8.83)\end{array}$ & 0.002 \\
\hline \multicolumn{6}{|l|}{ TB history } \\
\hline New & 326 & 296 & $30(9.2)$ & Ref & \\
\hline Retreatment & 56 & 50 & $6(10.7)$ & $\begin{array}{c}1.2 \\
(0.47-2.99)\end{array}$ & 0.721 \\
\hline $\mathrm{NA}^{*}$ & 3 & 2 & $1(33.3)$ & - & - \\
\hline \multicolumn{6}{|l|}{ Lineage } \\
\hline L2 (Beijing) & 56 & 52 & $4(7.1)$ & Ref & \\
\hline L1 (EAI) & 14 & 11 & $3(21.4)$ & $\begin{array}{c}3.5 \\
(0.69-18.1)\end{array}$ & 0.129 \\
\hline L3 (Delhi/CAS) & 99 & 89 & $10(10.1)$ & $\begin{array}{c}1.5 \\
(0.44-4.89)\end{array}$ & 0.539 \\
\hline L4 (Euro-American) & 215 & 196 & $19(8.8)$ & $\begin{array}{c}1.3 \\
(0.41-3.86)\end{array}$ & 0.868 \\
\hline M. bovis* & 1 & 0 & $1(100)$ & - & - \\
\hline Total & 385 & 348 & $37(9.6)$ & - & - \\
\hline
\end{tabular}

No. = number, $\mathrm{OR}=$ odds ratio, $\mathrm{CI}=$ confidence interval, $\mathrm{NA}=$ not available, $\mathrm{L}=$ lineage ${ }^{*}=$ variable (individuals with missing data) excluded from the regression model.

Overall, 30\% (6/20) of the MDR MTBC strains were identified with molecular resistant markers to all first-line drugs (Table S1). Furthermore, L3 (7/20, 35.0\%) and L4 (7/20, $35.0 \%$ ) equally dominated among the MDR TB strains. Comparison of the proportions of drug-resistant strains across the phylogenetic lineages (L1-L4) using logistic regression revealed no statistical differences (Table 3).

\subsection{Genome-Based Cluster Analysis}

We performed a genome-based cluster analysis with thresholds of a maximum genetic distance of $\leq 12$ and $\leq 5$ SNPs between any two MTBC strains to obtain an indication of potential TB transmission events [28,29]. Overall, 56.6\% (218/385, 95\% CI 51.6-61.5) of the MTBC strains were grouped into 61 genome clusters based on a $\leq 12$ SNP basis. The cluster size ranged between 2 and 28 strains / patients per cluster (Figure S3). If a stricter genetic 
distance of $\leq 5$ SNPs was used for cluster analysis, 30.4\% (117/385, 95\% CI 26.0-35.2) of the strains were grouped into 41 clusters ranging from 2 to 21 strains/patients (Figure S4).

Stratifying by phylogenetic MTBC lineage showed that L2 strains had the highest genome clustering rate using both $\leq 12$ and $\leq 5$ SNP distance $(66.1 \%$ and $35,7 \%)$, followed by L4 $(57.2 \%$ and $34,4 \%)$. L2 and L4 strains further had a higher odds ratio to be in a molecular cluster $(\mathrm{L} 2$, OR 2.1, $p$-value $=0.051$ and L4 OR 1.9, $p$-value $=0.019)$ using a SNP threshold of $\leq 5$ (Table 4). This indicates that L2 and L4 strains are more effectively transmitted as compared to L3 strains. However, a statistical difference was only observed for the strict threshold of $\leq 5$ SNP genetic distance (Table 4).

Table 4. Comparison of the proportions of clustered and not clustered strains across the detected MTBC phylogenetic lineages by using logistic regression in Nairobi, Kenya (2010-2015).

\begin{tabular}{|c|c|c|c|c|c|c|c|c|c|}
\hline \multirow{2}{*}{ Variable } & \multirow{2}{*}{ No. } & \multicolumn{4}{|c|}{ d12 } & \multicolumn{4}{|c|}{ d5 } \\
\hline & & NC & $C(\%)$ & OR $(95 \%$ CI) & $p$-Value & NC & C (\%) & OR $(95 \%$ CI $)$ & $p$-Value \\
\hline \multicolumn{10}{|l|}{ Lineage } \\
\hline L3 (Delhi/CAS) & 99 & 45 & $54(54.5 \%)$ & Ref & & 78 & $21(21.2 \%)$ & Ref & \\
\hline L2 (Beijing) & 56 & 19 & 37 (66.1\%) & $1.6(0.82-3.20)$ & 0.163 & 36 & $20(35.7 \%)$ & $2.1(1.00-4.28$ & 0.051 \\
\hline $\begin{array}{l}\text { L4 (Euro- } \\
\text { American) }\end{array}$ & 215 & 92 & $\begin{array}{c}123 \\
(57.2 \%)\end{array}$ & $1.1(0.69-1.80)$ & 0.658 & 141 & $74(34.4 \%)$ & $1.9(1.12-3.41)$ & 0.019 \\
\hline L1 (EAI) * & 14 & 10 & $4(28.6 \%)$ & - & - & 12 & $2(14.3 \%)$ & - & - \\
\hline M. bovis * & 1 & 0 & $0(0.00 \%)$ & - & - & 0 & $0(0.00 \%)$ & - & - \\
\hline Total & 385 & 166 & $218(56.6)$ & - & - & 267 & $117(30.5)$ & - & - \\
\hline
\end{tabular}

No. $=$ number, $\mathrm{d} 12=$ genetic distance of $\leq 12$ single-nucleotide polymorphisms (SNPs), $\mathrm{d} 5=$ genetic distance $\leq 5 \mathrm{SNPs}$, $\mathrm{NC}=$ number of not clustered, $\mathrm{C}=$ number of clustered, $\mathrm{OR}=$ odds ratio, $\mathrm{CI}=$ confidence interval, $\mathrm{NA}=$ not available, ${ }^{*}=$ variable excluded from the regression model.

Among the strains of the L4 MTBC sublineages, strains of the L4.4.1.1 (S-type) sublineage formed the largest genome cluster comprising 28 strains/patients. Overall, L4.4.1.1 (S-type) (OR 2.19, $p=0.001)$, L4.1.1.1 (X-type) (OR 2.7, $p=0.028)$, and L4.8 (Kenya H37Rvlike) (OR 2.12, $p=0.017)$ were significantly associated with molecular clusters.

\subsection{Transmission of MDR MTBC Strains}

Based on $\leq 12$ and $\leq 5$ SNP genetic distances, $60 \%$ (12/20, three clusters) and 50\% (10/20, three clusters) of MDR strains were clustered, respectively (Figure S5). Within the genomic clusters, MDR strains showed identical resistance-conferring mutations in the kat $G$ gene. In the $r p o B$ gene, most strains $(11 / 20,55.0 \%)$ carried the $r p o B$ mutation Ser450Leu as well as katG Ser315Thr (18/20, 90\%). The largest MDR cluster (with both thresholds, $\leq 12$ and $\leq 5$ SNPs) comprised six L4.4.1.1 (S-type) strains that all shared the following resistance-conferring mutations: katG Ser315Thr, rpoB Ser450Leu, pncA Lys96Thr, rpsL Lsy43Arg, and embB Met306Ile. One of the strains of this cluster already evolved to pre-XDR. All patients within this MDR cluster were from urban living areas in Nairobi.

\section{Discussion}

In this study, we elucidated the MTBC population structure and transmission events in Nairobi, Kenya, by using WGS. We showed that pulmonary TB is dominantly caused by strains of L4 and L3 and noted a substantial cluster rate which points towards ongoing transmission of MTBC strains. Moreover, all patients infected with MDR strains were from urban areas of Nairobi. We further found a cluster that consists of strains that are resistant to all first-line anti-TB drugs, with one of them already evolved to pre-XDR. These findings emphasize the need for more focused infection control measures and contact tracing of patients with MDR TB to break the transmission chains.

We revealed that the MTBC population comprises strains of four lineages in Nairobi, namely L1-L4 and one M. bovis strain. Strains of L4 and L3 are the main causative agents 
of TB comprising $55.8 \%$ and $25.7 \%$ of the investigated strains, respectively. These findings mirror reports from neighboring countries, such as Ethiopia [33], Tanzania [34], and Uganda [35] but are in contrast to the findings from Shuaib et al. [12] and Ejo et al. [36] in Eastern Sudan and Northwest Ethiopia where L3 strains were the main etiological agent of TB.

We found that strains of the LAM (L4.3.1, L4.3.3, and L4.3.4) sublineages are the most prevalent in Nairobi in comparison with strains of other L4 sublineages. Stucki et al. [37] have identified strains of certain sublineages of L4, such as LAM (L4.3), Haarlem (L4.1.2), and PGG3 (L4.10), as generalists as these strains can cause TB in all host genetic backgrounds globally. Therefore, the high prevalence of the strains of the generalist LAM sublineage might explain the dominance of L4 strains in Nairobi in Kenya. Another possible explanation for the success of L4 might be related to the observation that some L4 strains are capable of evading the host immune response and rapidly progressing to TB disease and thus are more often transmitted $[15,36]$.

As mentioned before, L3 strains were the second most prevalent causative agent of TB in Nairobi. It's been previously suggested that L3 strains have an evolutionary origin in South Asia. However, L3 strains have frequently been isolated from TB patients in East and North Africa [12,34,36,38], and one could speculate if these strains have coevolved with and adapted to their East African hosts and consequently developed specific biological/phenotypic traits in this particular host population [12]. The high prevalence of L3 strains in Kenya could also be related to recent and/or past movements of people from the Indian subcontinent (i.e., migration, tourism, and trade).

In this study, we found that L2 strains comprised $14.5 \%$ of the investigated strains, which is higher than former reports where the prevalence of L2 strains was 8.4\% in 2004 [39]. This upward trend indicates that strains of this lineage are also successfully transmitted in the population. Moreover, the reported proportion of L2 strains in the present study is higher than that in other countries of the region, including Sudan (0.6\%) [12], Tanzania (4.1\%) [34], Uganda (1.2\%) [38], and Ethiopia (0.5\%) [40]. It is known that L2 strains have their origin in East Asia, where they are responsible for multiple TB epidemics $[10,41]$. The expansion of L2 strains on the African continent has been suggested to be associated with increased transmission, high virulence, and a rapid progression to disease, rather than with drug resistance [10,41]. In addition, labor migration has been discussed as a possible factor explaining the increasing prevalence of L2 strains, mainly in East African countries [41].

We found evidence of direct patient-to-patient MTBC transmission as one-third (i.e., $30.4 \%$ ) of the isolates/patients were associated with genomic clusters using a $\leq 5$ SNP threshold in this study. Surprisingly, the transmission of TB is significantly higher in urban areas than in slum areas of Nairobi using both $\leq 12$ and $\leq 5$ SNP distances (34.9\% in urban areas vs. $24.7 \%$ in slum areas, $\operatorname{Exp}(B)=1.6, p$-value $=0.032$ ). This indicates that a higher risk of being infected due to transmission is related to a certain hotspot. Comparing the cluster rate across all phylogenetic lineages showed that L2 and L4 strains had an increased odds ratio of being in a molecular cluster (d5: L2, OR 2.1, $p$-value $=0.051$ and L4 OR 1.9, $p$-value $=0.019$ ). Additionally, among the strains of L4 MTBC sublineages, strains of the S-type, X-type, and Kenya H37Rv-like are likely more successful, i.e., have a higher odds to be in a molecular cluster. The observed cluster rate in Nairobi is comparable to the cluster rates reported in other high TB burden countries, such as Sudan [12], South Africa [16], and Ethiopia [42].

Early detection of resistance to anti-TB drugs is important for successful treatment and control of MDR TB [43]. In this study, $9.6 \%$ of the strains were found to be resistant to at least one first-line anti-TB drug, with 5.2\% being MDR strains. This proportion of MDR strains is higher than those previously reported in Kenya [44,45] and in other countries, e.g., Uganda (1.7\%) [46]. Nevertheless, in Sudan (9.0\%) [12] and Tanzania (6.3\%) [47], higher proportions of MDR strains have been reported. The variations in resistance rates observed in our study and the previous studies in Kenya could be due to the study design and sample size obtained [44,45]. Information on susceptibility patterns of strains against 
anti-TB drugs is essential for the control and surveillance of TB. Therefore, early diagnosis and treatment, improving treatment outcomes, and expanding diagnostic capacity for mycobacterial culture and drug susceptibility testing are crucial to limit the spread of drug resistant MTBC strains, especially MDR. In the case of the MDR and RR TB cases, rapid detection of resistance, e.g., by GeneXpert, followed by extended phenotypic drug susceptibility testing is essential for patient isolation and establishment of second-line anti-TB therapy. Otherwise, prolonged periods of ineffective treatment will likely allow continuous transmission of MDR cases with disastrous consequences for TB control in the future.

Furthermore, we found a high cluster rate among MDR strains $(60 \%$ based on $\leq 12$ SNP threshold and $50 \%$ based on $\leq 5$ SNP threshold) in combination with an increased risk of infections with drug-resistant strains in urban living areas in Nairobi. This is in line with a recent Kenya TB prevalence survey that indicated that the majority of TB cases are found in urban areas [14]. Therefore, successful control of TB in these settings should focus on addressing the influence of associated social and economic factors as well as strengthen TB control measures including contact tracing, early TB case detection, and adherence to treatment.

One limitation of our study is the lack of comprehensive epidemiological and clinical data, such as HIV status, diabetes, and other comorbidities, as well as treatment outcome. This limited a detailed analysis of epidemiological and clinical factors associated with recent transmission of MTBC strains (i.e., by using number of clustered strains as surrogate). Another limitation is the sampling bias of the two cohorts. Higher proportions of MDR and clustered strains were collected mainly from TB patients living in urban areas in the 2010 study cohort. This could have biased the association between any drug resistance and urban settings in the combined analysis.

\section{Conclusions}

Our study demonstrates that TB is predominantly caused by L4 strains in the urban and slum settings of Nairobi, Kenya. WGS analysis provides a better understanding of transmission dynamics of MTBC strains and their molecular drug resistance determinants. We found a cluster of strains that are resistant to all first-line anti-TB drugs. One of the strains of this molecular cluster has already evolved to a pre-XDR genotype via the acquisition of FQ resistance. Importantly, we observed that all MDR strains are associated with urban living environments and half of them are linked to direct patient-to-patient transmission, highlighting the urgent need to increase efforts to identify MDR TB cases and trace patient contacts to contain the spread of the disease in Nairobi. Adoption of the use of WGS may further allow for improved disease surveillance in high TB burden countries. Moreover, additional facilities in which molecular diagnosis of TB and drug susceptibility testing can be performed are required.

Supplementary Materials: The following supporting information can be downloaded at: https: / / www.mdpi.com/article/10.3390/genes13030475/s1, Figure S1. Sectional map of Nairobi, Kenya; Figure S2. Phylogenetic tree showing MTBC lineage classification of all analyzed MTBC isolates in urban and slum settings of Nairobi, Kenya; Figure S3. Phylogenetic tree showing $<12$ SNP clusters in urban and slum settings of Nairobi, Kenya; Figure S4. Phylogenetic tree showing $<5$ SNP clusters in urban and slum settings of Nairobi, Kenya; Figure S5. Phylogenetic tree showing genotypic drug resistance in urban and slum settings of Nairobi, Kenya; Table S1. Study Variables.

Author Contributions: Conceptualization, G.K., P.N., S.K. and S.N.; methodology, G.K., P.N. and Y.A.S.; software, S.H., S.K., M.M. and S.N.; validation, S.H., S.K., M.M. and S.N.; formal analysis, G.K., P.N. and Y.A.S.; investigation, G.K. and P.N.; resources, E.A., G.R., S.K. and S.N.; data curation, G.K., P.N., Y.A.S., S.H., S.K., M.M. and S.N.; writing-original draft preparation, G.K., Y.A.S. and M.M.; writing-review and editing, G.K., P.N., Y.A.S., E.A., G.R., S.H., S.K., M.M. and S.N.; visualization, G.K. and Y.A.S.; supervision, S.H., S.K., M.M. and S.N.; project administration, G.K. and P.N.; funding acquisition, G.K., P.N. and S.N. All authors have read and agreed to the published version of the manuscript. 
Funding: The first author (G.K.) is a DAAD (German: Deutscher Akademischer Austauschdienst) stipend recipient, funding program No. A/13/93800. Parts of this work have been supported by Deutsche Forschungsgemeinschaft (DFG, German Research Foundation) under Germany's Excellence Strategy EXC 22167-390884018, the Leibniz Science Campus EvoLUNG, and the German Center for Infection Research.

Institutional Review Board Statement: Ethical approval to conduct the study was granted by the Kenya Medical Research Institute Ethical Review Committee (No. KEMRI/RES/7/3/1).

Informed Consent Statement: Only pulmonary TB patients attending the TB clinic and who willingly consented to the study were recruited. In the case of illiterate individuals, consent was sought from their guardians.

Data Availability Statement: Generated raw sequencing reads in this study were submitted to the European Nucleotide Archive (ENA) under the project accession number (PRJEB50767).

Acknowledgments: The authors would like to thank all the patients who participated in this study as well as Edward Otieno and Lucy Nyang'au for helping with sample collection. We also thank the laboratory staff at the Molecular and Experimental Mycobacteriology Laboratory for their great assistance with the molecular work. In particular, we are grateful to Tanja Ubben, Julia Zallet, Tanja Struve-Sonnenschein, and Vanessa Mohr for providing excellent technical assistance.

Conflicts of Interest: The authors declare no conflict of interest.

\section{References}

1. WHO. Global Tuberculosis Report 2021; World Health Organization: Geneva, Switzerland, 2021; p. 232.

2. Glaziou, P.; Sismanidis, C.; Floyd, K.; Raviglione, M. Global epidemiology of tuberculosis. Cold Spring Harb. Perspect. Med. 2014, 5, a017798. [CrossRef] [PubMed]

3. Kipruto, H.; Mung'atu, J.; Ogila, K.; Adem, A.; Mwalili, S.; Masini, E.; Kibuchi, E. The epidemiology of tuberculosis in Kenya, a high TB HIV burden country (2000-2013). Int. J. Public Health Epidemiol. Res. 2015, 1, 002-013.

4. Kwan, C.K.; Ernst, J.D. HIV and tuberculosis: A deadly human syndemic. Clin. Microbiol. Rev. 2011, 24, 351-376. [CrossRef] [PubMed]

5. $\quad$ Campbell, P.J.; Morlock, G.P.; Sikes, R.D.; Dalton, T.L.; Metchock, B.; Starks, A.M.; Hooks, D.P.; Cowan, L.S.; Plikaytis, B.B.; Posey, J.E. Molecular detection of mutations associated with first- and second-line drug resistance compared with conventional drug susceptibility testing of Mycobacterium tuberculosis. Antimicrob. Agents Chemother. 2011, 55, 2032-2041. [CrossRef]

6. Cilloni, L.; Fu, H.; Vesga, J.F.; Dowdy, D.; Pretorius, C.; Ahmedov, S.; Nair, S.A.; Mosneaga, A.; Masini, E.; Sahu, S.; et al. The potential impact of the COVID-19 pandemic on the tuberculosis epidemic a modelling analysis. EClinicalMedicine 2020, $28,100603$. [CrossRef]

7. McQuaid, C.F.; Vassall, A.; Cohen, T.; Fiekert, K.; White, R.G. The impact of COVID-19 on TB: A review of the data. Int. J. Tuberc. Lung Dis. 2021, 25, 436-446. [CrossRef]

8. Singhal, R.; Myneedu, V.P. Microscopy as a diagnostic tool in pulmonary tuberculosis. Int J. Mycobacteriol. 2015, 4, 1-6. [CrossRef]

9. Shuaib, Y.A.; Khalil, E.A.G.; Schaible, U.E.; Wieler, L.H.; Bakheit, M.A.M.; Mohamed-Noor, S.E.; Abdalla, M.A.; Homolka, S.; Andres, S.; Hillemann, D.; et al. Smear Microscopy for Diagnosis of Pulmonary Tuberculosis in Eastern Sudan. Tuberc. Res. Treat. 2018, 2018, 8038137. [CrossRef]

10. Merker, M.; Blin, C.; Mona, S.; Duforet-Frebourg, N.; Lecher, S.; Willery, E.; Blum, M.G.; Rusch-Gerdes, S.; Mokrousov, I.; Aleksic, E.; et al. Evolutionary history and global spread of the Mycobacterium tuberculosis Beijing lineage. Nat. Genet. 2015, 47, 242-249. [CrossRef]

11. Merker, M.; Barbier, M.; Cox, H.; Rasigade, J.P.; Feuerriegel, S.; Kohl, T.A.; Diel, R.; Borrell, S.; Gagneux, S.; Nikolayevskyy, V.; et al. Compensatory evolution drives multidrug-resistant tuberculosis in Central Asia. Elife 2018, 7. [CrossRef]

12. Shuaib, Y.A.; Khalil, E.A.G.; Wieler, L.H.; Schaible, U.E.; Bakheit, M.A.; Mohamed-Noor, S.E.; Abdalla, M.A.; Kerubo, G.; Andres, S.; Hillemann, D.; et al. Mycobacterium tuberculosis Complex Lineage 3 as Causative Agent of Pulmonary Tuberculosis, Eastern Sudan. Emerg. Infect. Dis. 2020, 26, 427-436. [CrossRef] [PubMed]

13. Kompala, T.; Shenoi, S.V.; Friedland, G. Transmission of tuberculosis in resource-limited settings. Curr. HIV/AIDS Rep. 2013, 10, 264-272. [CrossRef] [PubMed]

14. Enos, M.; Sitienei, J.; Ong'ang'o, J.; Mungai, B.; Kamene, M.; Wambugu, J.; Kipruto, H.; Manduku, V.; Mburu, J.; Nyaboke, D.; et al. Kenya tuberculosis prevalence survey 2016: Challenges and opportunities of ending TB in Kenya. PLoS ONE 2018, 13, e0209098. [CrossRef] [PubMed]

15. Wiens, K.E.; Woyczynski, L.P.; Ledesma, J.R.; Ross, J.M.; Zenteno-Cuevas, R.; Goodridge, A.; Ullah, I.; Mathema, B.; Djoba Siawaya, J.F.; Biehl, M.H.; et al. Global variation in bacterial strains that cause tuberculosis disease: A systematic review and meta-analysis. BMC Med. 2018, 16, 196. [CrossRef] 
16. Middelkoop, K.; Mathema, B.; Myer, L.; Shashkina, E.; Whitelaw, A.; Kaplan, G.; Kreiswirth, B.; Wood, R.; Bekker, L.G. Transmission of tuberculosis in a South African community with a high prevalence of HIV infection. J. Infect. Dis. 2015, 211, 53-61. [CrossRef]

17. Ganoza, C.A.; Ricaldi, J.N.; Chauca, J.; Rojas, G.; Munayco, C.; Agapito, J.; Palomino, J.C.; Guerra, H. Novel hypertonic salinesodium hydroxide (HS-SH) method for decontamination and concentration of sputum samples for Mycobacterium tuberculosis microscopy and culture. J. Med. Microbiol. 2008, 57, 1094-1098. [CrossRef]

18. Basavaraj, A.; Chandanwale, A.; Patil, A.; Kadam, D.; Joshi, S.; Gupte, N.; McIntire, K.; Jain, D.; Dalal, H.; Badave, R.; et al Tuberculosis Risk among Medical Trainees, Pune, India. Emerg. Infect. Dis. 2016, 22, 541-543. [CrossRef]

19. GLI. Mycobacteriology Laboratory Manual; WHO: Geneva, Swizerland, 2014.

20. Somerville, W.; Thibert, L.; Schwartzman, K.; Behr, M.A. Extraction of Mycobacterium tuberculosis DNA: A question of containment. J. Clin. Microbiol. 2005, 43, 2996-2997. [CrossRef]

21. Baym, M.; Kryazhimskiy, S.; Lieberman, T.D.; Chung, H.; Desai, M.M.; Kishony, R. Inexpensive multiplexed library preparation for megabase-sized genomes. PLoS ONE 2015, 10, e0128036. [CrossRef]

22. Li, H.; Durbin, R. Fast and accurate long-read alignment with Burrows-Wheeler transform. Bioinformatics 2010, 26, 589-595. [CrossRef]

23. Kohl, T.A.; Utpatel, C.; Schleusener, V.; De Filippo, M.R.; Beckert, P.; Cirillo, D.M.; Niemann, S. MTBseq: A comprehensive pipeline for whole genome sequence analysis of Mycobacterium tuberculosis complex isolates. Peer] 2018, 6, e5895. [CrossRef] [PubMed]

24. Feuerriegel, S.; Schleusener, V.; Beckert, P.; Kohl, T.A.; Miotto, P.; Cirillo, D.M.; Cabibbe, A.M.; Niemann, S.; Fellenberg, K. PhyResSE: A Web Tool Delineating Mycobacterium tuberculosis Antibiotic Resistance and Lineage from Whole-Genome Sequencing Data. J. Clin. Microbiol. 2015, 53, 1908-1914. [CrossRef] [PubMed]

25. Posada, D. jModelTest: Phylogenetic model averaging. Mol. Biol. Evol. 2008, 25, 1253-1256. [CrossRef] [PubMed]

26. Price, M.N.; Dehal, P.S.; Arkin, A.P. FastTree 2-approximately maximum-likelihood trees for large alignments. PLoS ONE 2010, 5, e9490. [CrossRef]

27. He, Z.; Zhang, H.; Gao, S.; Lercher, M.J.; Chen, W.H.; Hu, S. Evolview v2: An online visualization and management tool for customized and annotated phylogenetic trees. Nucleic Acids Res. 2016, 44, W236-W241. [CrossRef]

28. Walker, T.M.; Ip, C.L.C.; Harrell, R.H.; Evans, J.T.; Kapatai, G.; Dedicoat, M.J.; Eyre, D.W.; Wilson, D.J.; Hawkey, P.M.; Crook, D.W.; et al. Whole-genome sequencing to delineate Mycobacterium tuberculosis outbreaks: A retrospective observational study. Lancet Infect. Dis. 2013, 13, 137-146. [CrossRef]

29. Meehan, C.J.; Moris, P.; Kohl, T.A.; Pecerska, J.; Akter, S.; Merker, M.; Utpatel, C.; Beckert, P.; Gehre, F.; Lempens, P.; et al. The relationship between transmission time and clustering methods in Mycobacterium tuberculosis epidemiology. EBioMedicine 2018, 37, 410-416. [CrossRef]

30. MARCH. DLTLD Guidelines on Management of Leprosy and Tuberculosis; Ministry of Public Health and Sanitation: Nairobi, Kenya, 2009; p. 108.

31. Coll, F.; McNerney, R.; Guerra-Assuncao, J.A.; Glynn, J.R.; Perdigao, J.; Viveiros, M.; Portugal, I.; Pain, A.; Martin, N.; Clark, T.G. A robust SNP barcode for typing Mycobacterium tuberculosis complex strains. Nat. Commun. 2014, 5, 4812. [CrossRef]

32. Niemann, S.; Merker, M.; Kohl, T.; Supply, P. Impact of Genetic Diversity on the Biology of Mycobacterium tuberculosis Complex Strains. Microbiol. Spectr. 2016, 4, 1-18. [CrossRef]

33. Nuru, A.; Mamo, G.; Worku, A.; Admasu, A.; Medhin, G.; Pieper, R.; Ameni, G. Genetic Diversity of Mycobacterium tuberculosis Complex Isolated from Tuberculosis Patients in Bahir Dar City and Its Surroundings, Northwest Ethiopia. Biomed. Res. Int. 2015, 2015, 174732. [CrossRef]

34. Mbugi, E.V.; Katale, B.Z.; Siame, K.K.; Keyyu, J.D.; Kendall, S.L.; Dockrell, H.M.; Streicher, E.M.; Michel, A.L.; Rweyemamu, M.M.; Warren, R.M.; et al. Genetic diversity of Mycobacterium tuberculosis isolated from tuberculosis patients in the Serengeti ecosystem in Tanzania. Tuberculosis 2015, 95, 170-178. [CrossRef] [PubMed]

35. Asiimwe, B.B.; Ghebremichael, S.; Kallenius, G.; Koivula, T.; Joloba, M.L. Mycobacterium tuberculosis spoligotypes and drug susceptibility pattern of isolates from tuberculosis patients in peri-urban Kampala, Uganda. BMC Infect. Dis. 2008, 8, 101. [CrossRef] [PubMed]

36. Ejo, M.; Torrea, G.; Uwizeye, C.; Kassa, M.; Girma, Y.; Bekele, T.; Ademe, Y.; Diro, E.; Gehre, F.; Rigouts, L.; et al. Genetic diversity of the Mycobacterium tuberculosis complex s.strains from newly diagnosed tuberculosis patients in Northwest Ethiopia reveals a predominance of East-African-Indian and Euro-American lineages. Int. J. Infect. Dis. 2021, 103, 72-80. [CrossRef] [PubMed]

37. Stucki, D.; Brites, D.; Jeljeli, L.; Coscolla, M.; Liu, Q.; Trauner, A.; Fenner, L.; Rutaihwa, L.; Borrell, S.; Luo, T.; et al. Mycobacterium tuberculosis lineage 4 comprises globally distributed and geographically restricted sublineages. Nat. Genet. 2016, 48, 1535-1543. [CrossRef]

38. Bazira, J.; Asiimwe, B.B.; Joloba, M.L.; Bwanga, F.; Matee, M.I. Mycobacterium tuberculosis spoligotypes and drug susceptibility pattern of isolates from tuberculosis patients in South-Western Uganda. BMC Infect. Dis. 2011, 11, 81. [CrossRef]

39. Githui, W.A.; Jordaan, A.M.; Juma, E.S.; Kinyanjui, P.; Karimi, F.G.; Kimwomi, J.; Meme, H.; Mumbi, P.; Streicher, E.M.; Warren, R.; et al. Identification of MDR-TB BeijingW and other Mycobacterium tuberculosis genotypes in Nairobi, Kenya. Int J. Tuberc. Lung Dis. 2004, 8, 352-360. 
40. Yimer, S.A.; Norheim, G.; Namouchi, A.; Zegeye, E.D.; Kinander, W.; Tonjum, T.; Bekele, S.; Mannsaker, T.; Bjune, G.; Aseffa A.; et al. Mycobacterium tuberculosis lineage 7 strains are associated with prolonged patient delay in seeking treatment for pulmonary tuberculosis in Amhara Region, Ethiopia. J. Clin. Microbiol. 2015, 53, 1301-1309. [CrossRef]

41. Rutaihwa, L.K.; Menardo, F.; Stucki, D.; Gygli, S.M.; Ley, S.D.; Malla, B.; Feldmann, J.; Borrell, S.; Beisel, C.; Middelkoop, K.; et al. Multiple Introductions of Mycobacterium tuberculosis Lineage 2-Beijing Into Africa Over Centuries. Front. Ecol. Evol. 2019, 7, 112. [CrossRef]

42. Mekonnen, A.; Merker, M.; Collins, J.M.; Addise, D.; Aseffa, A.; Petros, B.; Ameni, G.; Niemann, S. Molecular epidemiology and drug resistance patterns of Mycobacterium tuberculosis complex isolates from university students and the local community in Eastern Ethiopia. PLoS ONE 2018, 13, e0198054. [CrossRef]

43. Cain, K.P.; Marano, N.; Kamene, M.; Sitienei, J.; Mukherjee, S.; Galev, A.; Burton, J.; Nasibov, O.; Kioko, J.; De Cock, K.M. The movement of multidrug-resistant tuberculosis across borders in East Africa needs a regional and global solution. PLoS Med. 2015, 12, e1001791. [CrossRef]

44. Ogaro, T.D.; Githui, W.; Kikuvi, G.; Okari, J.; Wangui, E.; Asiko, V. Anti-tuberculosis drug resistance in Nairobi, Kenya. African J. Health Sci. 2012, 20, 21-27.

45. Obonyo, L.; Amukoye, E.; Zipporah, P. First line anti-tuberculosis drug resistance among human immunodeficiency virus infected patients attending Maryland Comprehensive Care Centre, Mathare 4a, Nairobi, Kenya. Int. J. Sci. Basic Appl. Res. 2014, 15, 661-668.

46. Kateregga, N.; Atuheire, C.; Ntambi, S.; Ocaka, D.; Ndukui, G.J.; Wampande, E. Prevalence of multidrug resistant Mycobacterium tuberculosis and risk factors among youth attending MDR-TB unit in Mulago hospital. J. Bacteriol. Mycol. 2018, 5, 1088.

47. Hoza, A.S.; Mfinanga, S.G.M.; Konig, B. Anti-TB drug resistance in Tanga, Tanzania: A cross sectional facility-base prevalence among pulmonary TB patients. Asian Pac. J. Trop. Med. 2015, 8, 907-913. [CrossRef] 